Journal of

Synchrotron

Radiation

ISSN 0909-0495

Received 17 October 2011

Accepted 15 February 2012

\section{Performance upgrade in the JAEA actinide science beamline BL23SU at SPring-8 with a new twin-helical undulator}

\author{
Yuji Saitoh, ${ }^{\text {a* }}$ Yoshihiro Fukuda, ${ }^{a}$ Yukiharu Takeda, ${ }^{a}$ Hiroshi Yamagami, \\ Sunao Takahashi, ${ }^{c}$ Yoshihiro Asanoo, ${ }^{\mathrm{d}}$ Toru Hara, ${ }^{\mathrm{d}}$ Katsutoshi Shirasawa, \\ Masao Takeuchi, ${ }^{\mathrm{c}}$ Takashi Tanaka ${ }^{\mathrm{d}, \mathrm{c}}$ and Hideo Kitamura ${ }^{\mathrm{d}}$ \\ ${ }^{a}$ Quantum Beam Science Directorate, Japan Atomic Energy Agency, 1-1-1 Kouto, Sayo, Hyogo \\ 679-5148, Japan, bepartment of Physics, Kyoto Sangyo University, Kyoto 603-8555, Japan, 'Japan \\ Synchrotron Radiation Research Institute, 1-1-1 Kouto, Sayo, Hyogo 679-5198, Japan, and dRIKEN \\ Harima Institute, 1-1-1 Kouto, Sayo, Hyogo 679-5148, Japan. E-mail: ysaitoh@spring8.or.jp
}

Keywords: twin-helical undulator; helicity switching; soft X-ray beamline; $X$-ray magnetic circular dichroism; uranium compounds.

\section{Introduction}

The JAEA actinide science beamline, BL23SU, built and operated by Japan Atomic Energy Agency (JAEA), is a soft X-ray beamline at the SPring-8 synchrotron facility with its first light being received in 1998 (Saitoh et al., 2001). The primary aim of BL23SU is to investigate the electronic structures of uranium-based compounds and related materials using soft X-ray photoemission (SX-PES) and magnetic circular dichroism (XMCD) spectroscopies with a focus on superconductivity and magnetism. The uranium compounds display a variety of intriguing phenomena, which is considered to be due to complex many-body interactions between the valence-electron states. Recently, superconductivity has been discovered in heavy-fermion ferromagnets, such as $\mathrm{UGe}_{2}$, URhGe, UCoGe and UIr [for a recent review see, for example, Pfleiderer (2009)], and therefore XMCD as well as SX-PES studies are required for a comprehensive understanding of the nature of the $5 f$ electrons. With the availability of synchrotron radiation sources, XMCD has been developing as a probe of local (element- and angular-momentum-specific) spin and orbital magnetic moments (Funk et al., 2005).

The BL23SU beamline was initially commissioned with an APPLE-2-type undulator with flexible polarization properties (Sasaki, 1994), in which the four rows of periodic magnets are arranged with two rows above and two rows below the stored electron orbit plane. The length $(L)$ of the undulator was $2 \mathrm{~m}$ and located at the upstream half of a $5.7 \mathrm{~m}$ straight section. The periodic length $\left(\lambda_{\mathrm{u}}\right)$ of the undulator was $12 \mathrm{~cm}$ and the number of periods $\left(N_{\mathrm{p}}\right)$ was 16 . It covered the energy range down to $240 \mathrm{eV}$ in circular polarization mode with a minimum gap of $25 \mathrm{~mm}$ (see Fig. 1). In this device, photon helicity was switched by the longitudinal mechanical shift of the magnetic arrays (i.e. changing the magnetic row phase), and the helicity switching at each photon energy of an XMCD spectrum was an important technical approach for high-accuracy XMCD experiments (Agui et al., 2001). A local feed-forward correction system for the orbit distortion at the switching time up to around $2.5 \mathrm{~s}$ between opposite helical polarizations (the phase parameter in its pure helical mode was roughly proportional to the gap) has been developed (Nakatani et al., 2005). This system allowed us to perform efficient XMCD measurements using a superconducting magnet under fixed sample conditions (Okamoto et al., 2004). This superconducting magnet requires a long-field reversing time and therefore point-bypoint field reversal was impractical. The frequent mechanical motion of the magnet arrays, however, resulted in a high percentage of beamline downtime caused by component failures, thus creating some problems with maintenance and parts replacement. With regard to the SX-PES experiments, a clear advantage of the circular polarization mode over the linear polarization mode was the higher stability in photon energy and intensity, reflecting the much lower heat load on optical components (Kincaid, 1977).

Meanwhile, the twin-helical undulator of BL25SU (Hara et al., 1998), ID25, pioneered at SPring-8, has provided reliable and productive operations for helicity-switching dichroism experiments (Muro et al., 2005; Nakamura et al., 2005; Hayashi 


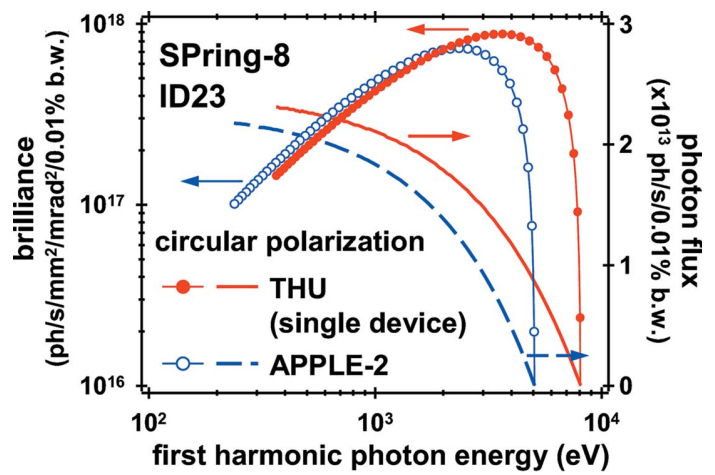

Figure 1

Source brilliance and photon flux in circular polarization mode calculated for the single device of the twin-helical undulator (THU) and the replaced APPLE-2 device of ID23 for SPring-8 $100 \mathrm{~mA}$ operation.

et al., 2011) with almost complete circular polarization $\left(P_{\mathrm{c}}\right)$ and full polarization reversal (Hirono et al., 2005) for a switching frequency up to $10 \mathrm{~Hz}$ (Shirasawa et al., 2004). The twin-helical undulator consists of two in-line helical undulators and five kicker magnets. In these helical undulators the six rows of periodic magnets are arranged with three rows above and three rows below the electron orbit plane. Each of the helical undulators is an out-of-vacuum device $\left(\lambda_{\mathrm{u}}=12 \mathrm{~cm}, N_{\mathrm{p}}=\right.$ 12) with a minimum gap of $20 \mathrm{~mm}$ to cover the energy range down to $120 \mathrm{eV}$. In helicity-switching mode, the kicker magnets alternately separate the photon beams horizontally by $0.3 \mathrm{mrad}$ in order to minimize the overlap between the cones of fundamental radiation with opposite helicities from the two devices. Coordinated scanning of the undulator gaps and the monochromator has also been successfully demonstrated in this mode. The angular aperture for the central radiation cones along the beamline axis is defined by front-end $X Y$ slits (Oura et al., 1998). A detailed description of the helicity switching in the twin-helical undulator has been given previously (Hara et al., 2003).

This type of undulator was adopted for BL23SU using invacuum helical undulators, each with $N_{\mathrm{p}}=17$, of $\lambda_{\mathrm{u}}=7.52 \mathrm{~cm}$ in order to enhance the photon flux above the $\mathrm{N} 1 s$ absorption edge. Fig. 1 shows a comparison of the brilliance and photon flux in circular polarization mode between the replaced APPLE-2 undulator $(L=2 \mathrm{~m})$ and the single new undulator $(L=1.3 \mathrm{~m})$ calculated using the program SPECTRA (Tanaka \& Kitamura, 2001). Unlike ID25, the central row of the magnetic array, namely the phase of the magnetic field, is fixed in ID23 because of the difficulty of having linear guide systems in an UHV environment. Thus the polarization of the soft $\mathrm{X}$-ray radiation was restricted to be circular. The two undulator gaps of the new ID23 can be independently adjusted down to $8 \mathrm{~mm}$ at present, covering photon energies down to $370 \mathrm{eV}$. Small gaps of the in-vacuum undulator allow us to reduce $\lambda_{\mathrm{u}}$, thereby increasing $N_{\mathrm{p}}$, being proportional to the photon flux output.

The maximum frequency of helicity switching is $10 \mathrm{~Hz}$, which is limited by the diagnostics of the electron beam orbit. To maintain coexistence with other beamlines, the variation of the electron beam orbit owing to helicity switching is required to be less than $\pm 1 \mu \mathrm{m}$ r.m.s. Since the operation of the kicker magnets is independent of the undulator gaps, a simple feedforward correction scheme is employed for the orbit correction. The twin-helical undulator of ID23 can also be applied to XMCD experiments at much higher switching frequency by using a fast mechanical chopper and angularly separated dual photon beams, in which the kicker magnets generate a stationary orbit bump (Sawhney et al., 1997).

The use of an electromagnetic undulator (Freeland et al., 2002) has an important advantage over the twin-helical undulator for an identical source point of both helicities. However, a fast orbit correction feed-back, which is currently not adopted in the SPring- 8 storage ring, is necessary, otherwise feed-forward tables should be made for each electromagnet current and the orbit correction becomes too complicated and less accurate (Oura et al., 2007). Therefore it was not considered as a new ID23.

Soft X-ray magnetic linear dichroism (XMLD) spectroscopy measurements were not included in the scientific case of BL23SU because both the experiment and interpretation were considered to be more challenging than for XMCD. For this reason, no XMLD experiments had been conducted with the APPLE-2 device.

Recently, the helicity switching capability at a frequency of $1 \mathrm{~Hz}$ has reached operational status as a first step. In this operation each of the two polarized beams is alternately supplied to the beamline for a period of $0.3 \mathrm{~s}$ with a transient time of $0.2 \mathrm{~s}$. We report here its performance for XMCD experiments as well as some recent upgrades of BL23SU.

\section{Beamline description}

A schematic overview of the main beamline components of BL23SU is shown in Fig. 2. The undulator radiation is transported by the front-end within the storage-ring shield wall to the downstream optics. In order to stop the off-axis beam deflected by the kicker magnets, some of the front-end components upstream of the $X Y$ slits, including heatabsorbing photon masks, have been replaced by ones with wider acceptances.

The optical system of BL23SU, as detailed elsewhere (Saitoh et al., 2001), consists of prefocusing mirrors ( $\mathrm{M}_{\mathrm{v}}$ and $\mathrm{M}_{\mathrm{h}}$ ) and a varied-line-spacing plane-grating monochromator (VLSPGM, from $S_{1}$ to $S_{2}$ ) equipped with an entrance slit and refocusing mirrors $\left(\mathrm{M}_{3 \mathrm{a}, \mathrm{b}}, \mathrm{M}_{3.5}, \mathrm{M}_{4 \mathrm{a}, \mathrm{b}}\right)$. This undulator replacement did not need the modification of a hutch enclosure housing the prefocusing mirrors. The VLSPGM was designed to provide an operational range from 0.2 to at least $1.5 \mathrm{keV}$ with mechanically ruled blazed gratings and achieved a resolving power in excess of $1 \times 10^{4}$ at the nitrogen $K$-edge in full aperture operation for the circular polarization mode of the APPLE-2 undulator (Saitoh et al., 2001). However, the photon flux available turned out to be insufficient for soft X-ray angle-resolved photoemission spectroscopy (SXARPES) successfully demonstrated at the BL25SU beamline (Suga et al., 2004). Recent SX-ARPES measurements at BL23SU with energy resolutions down to $0.1 \mathrm{eV}$ (Fujimori et 


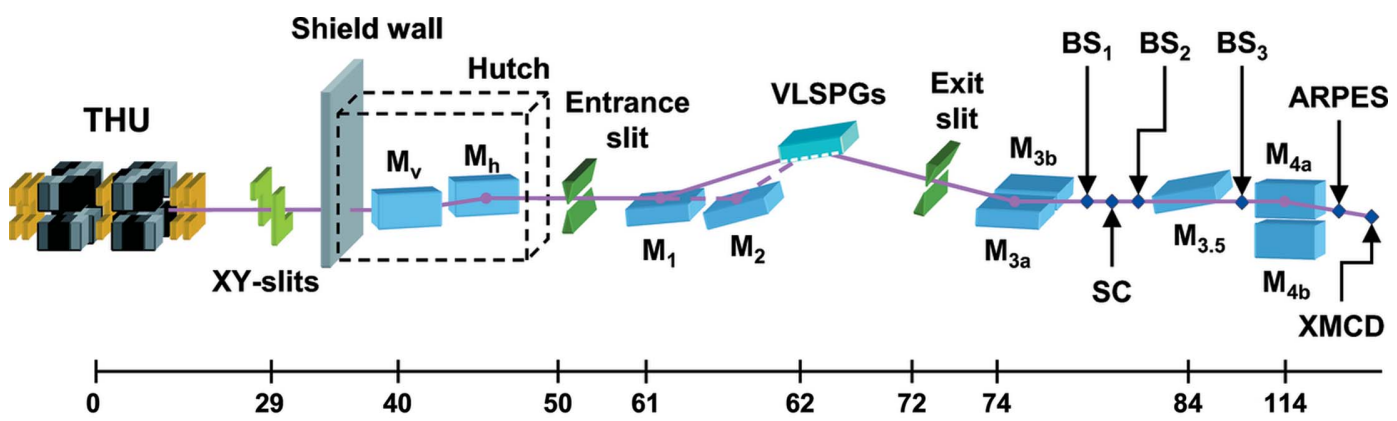

Figure 2

Schematic of the BL23SU beamline at SPring-8. The scale indicates the distance in meters from the center of the twin-helical undulator (THU). $\mathrm{M}_{\mathrm{v}}$ : vertical condensing mirror; $\mathrm{M}_{\mathrm{h}}$ : horizontal collimating mirror; $\mathrm{M}_{1}$ and $\mathrm{M}_{2}$ : vertical focusing mirrors; $\mathrm{M}_{3 \mathrm{a}}$ : vertical collimating mirror; $\mathrm{M}_{3 \mathrm{~b}}, \mathrm{M}_{3.5}, \mathrm{M}_{4 \mathrm{a}}$ and $\mathrm{M}_{4 \mathrm{~b}}$ : refocusing toroidal mirrors; $\mathrm{BS}_{1}-\mathrm{BS}_{3}$ : biophysical spectroscopy stations; SC: surface chemistry station. The $\mathrm{M}_{3 \mathrm{~b}}$ and $\mathrm{M}_{3.5}$ mirrors are used for experiments at the $\mathrm{SC}$ and $\mathrm{BS}_{3}$ stations, respectively.

al., 2007) have been facilitated by the substitution of a holographic ruled grating with a central groove density of 600 lines $\mathrm{mm}^{-1}$ into the VLSPGM, which resulted in practically fourfold improvement in throughput while providing a resolving power better than $1 \times 10^{4}$ up to $1 \mathrm{keV}$.

After the installation of the new twin-helical undulator, the optical components were slightly adjusted to accommodate a change in the source centers by $1 \mathrm{~m}$ by maximizing the transmitted flux at the highest resolution of the VLSPGM using both undulator beams. In this case, monochromatic flux was almost twice that of the APPLE-2 device, as expected, achieving $\sim 1 \times 10^{12}$ photons $\mathrm{s}^{-1}(0.01 \% \text { bandwidth })^{-1}$ at $700 \mathrm{eV}$ (see Fig. 3). The enhanced flux is quite useful, not only for SX-ARPES measurements (Kawasaki et al., 2011) but also for surface reaction analyses at the SC station (Teraoka \& Yoshigoe, 2001) and biological spectroscopies at the $\mathrm{BS}_{1}-\mathrm{BS}_{3}$ stations (Fujii et al., 2009; Yokoya \& Akamatsu, 2001; Ukai et al., 2009).

More recently, the SX-ARPES analyzer of a Scienta SES 2002 has been calibrated by modifying the voltage tables in a collaborative research with the Osaka University group of Sekiyama, Kiss et al. This calibration provided its original specification in angular resolution and effective angular window, which is accompanied by an enhancement in transmission and detection efficiency by a factor of at least six, thereby reducing data collection times and increasing throughput (Sekiyama et al., 2012).

The XMCD endstation terminates BL23SU at approximately $120 \mathrm{~m}$ from the light source. The experimental UHV chamber is equipped with the superconducting magnet and a sample holder attached to a liquid-He cryostat reaching a sample temperature of $4.5 \mathrm{~K}$. This magnet provides a variable field parallel to the photon beam direction in the range of $\pm 10 \mathrm{~T}$ with a sweeping rate up to $1 \mathrm{~T} \mathrm{~min}^{-1}$. XMCD spectra are obtained in total electron yield mode by measuring the sample drain current $(I)$ normalized by incident photon flux $\left(I_{0}\right)$ monitored with a Au-coated $\mathrm{SiO}_{2}$ refocusing toroidal mirror, $\mathrm{M}_{4 \mathrm{a}}$, or a Au-coated mesh between the $\mathrm{M}_{4 \mathrm{a}}$ mirror and the XMCD station. Each of the signals $I_{0}$ and $I$ is converted by a current amplifier into a voltage which is subsequently converted to a frequency. These signals are fed into separate counters for individual polarization. In the helicity-switching mode the signals are integrated during time periods determined by gating TTL (transistor-transistor logic) signals from the undulator control system. In addition to this, without the reconnection of signal cables, on-the-fly scanning mode (constant velocity motion of the VLSPG and either undulator gap) with a typical rate of $40 \mathrm{eV} \mathrm{min}^{-1}$ has been implemented (Takeda et al., 2008). This scanning mode was not effective for the APPLE-2 undulator, because the gap scan had been limited to a phase parameter of $0 \mathrm{~mm}$, corresponding to the horizontal linear polarization mode.

\section{Experimental observations}

Fig. 3 shows the photon flux measured at the XMCD endstation during the $1 \mathrm{~Hz}$-switching mode for a photon energy $(h v)$ of $700 \mathrm{eV}$ along with the received gating TTL signals for left- and right-handed circularly polarized (LCP and RCP) light. The beam-defining aperture, located at approximately $29 \mathrm{~m}$ from the center of the light source, was unchanged from $2.5 \mathrm{~mm} \times 2 \mathrm{~mm}$ (horizontally $\times$ vertically) that had been determined for SX-ARPES experiments. Both of the helical undulators were set to the same gap to maximize the photon flux. During the switching transients, both of the beams with opposite helicities pass through the beamline. The change in intensity of approximately $10 \%$ for each helicity, which was measured to be fairly independent of the first-

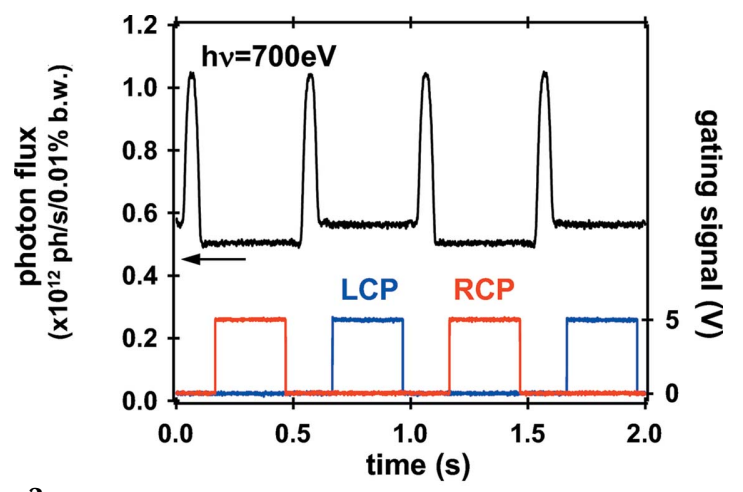

Figure 3

Photon flux during the helicity-switching mode measured for $h v=700 \mathrm{eV}$ at the XMCD station together with the gating TTL signals for the experiments. 


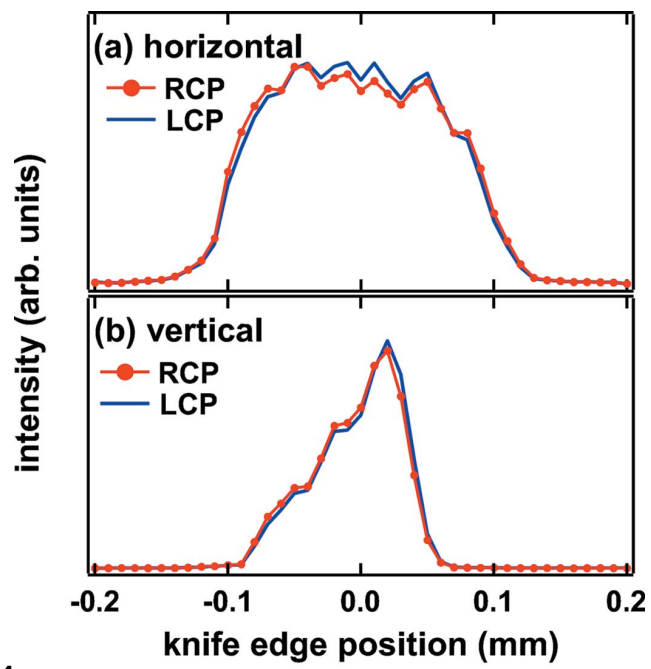

Figure 4

Horizontal $(a)$ and vertical $(b)$ beam spot profiles in the helicity-switching mode at $h v=700 \mathrm{eV}$ measured at the XMCD station.

harmonic photon energies, is ascribed to the difference in angular acceptance of the beam-defining aperture owing to the positional difference of $2 \mathrm{~m}$ between the two undulator centers. This is because the calculated flux transmitted through the aperture using the SPECTRA program adequately accounts for these behaviors.

Fig. 4 shows the intensity profiles of the focused beam spots at the XMCD station obtained at $h v=700 \mathrm{eV}$ while varying either vertical or horizontal knife-edge position. The focus spots are produced by the refocusing toroidal $\mathrm{M}_{4 \mathrm{a}}$ mirror which imaged the light source (exit slit width) in the horizontal (vertical) direction with a nominal demagnification of about 6 (1/3). The width of the VLSPGM exit slit was set to $20 \mu \mathrm{m}$. These measurements were conducted with the same beam intensity by detuning the downstream LCP undulator gap slightly. The LCP and RCP beams are focused on almost a common spot without additional optical alignment. The subtle difference has virtually no influence on dichroic experiments as shown below.

The XMCD performance was tested on a polycrystalline $\mathrm{Fe}$ (purity $99.99 \%$ ) sample at room temperature. Fig. 5 shows the photon-flux-normalized Fe $L_{2,3}$ XAS $\left(\mu_{\mathrm{L}}+\mu_{\mathrm{R}}\right)$ and XCD $\left(\mu_{\mathrm{L}}-\mu_{\mathrm{R}}\right)$ spectra measured with an energy resolution of $70 \mathrm{meV}$ for a fixed magnetic field of $6 \mathrm{~T}$, in which both helicities in one cycle were used for every energy point. Here, $\mu_{\mathrm{L}}$ $\left(\mu_{\mathrm{R}}\right)$ is the X-ray absorption for LCP (RCP) light. The $L_{3}$ $\mathrm{XAS}$ intensity from the pre-edge background is normalized to unity for ease of comparison with the results obtained by Chen et al. (1995) as described below. The noise level of the measured XMCD spectrum is approximately $\pm 0.03 \%$ of the XAS intensity, corresponding to an order of magnitude improvement in sensitivity in our beamline. This sensitivity permits the XMCD onset to be observed at about $18 \mathrm{eV}$ below the $L_{3}$ edge.

A closer look at the XMCD data by use of the APPLE-2 device shows that the XMCD sensitivity was generally limited by uneven signals taken immediately following the helicity switching performed at every two measurement points (Agui

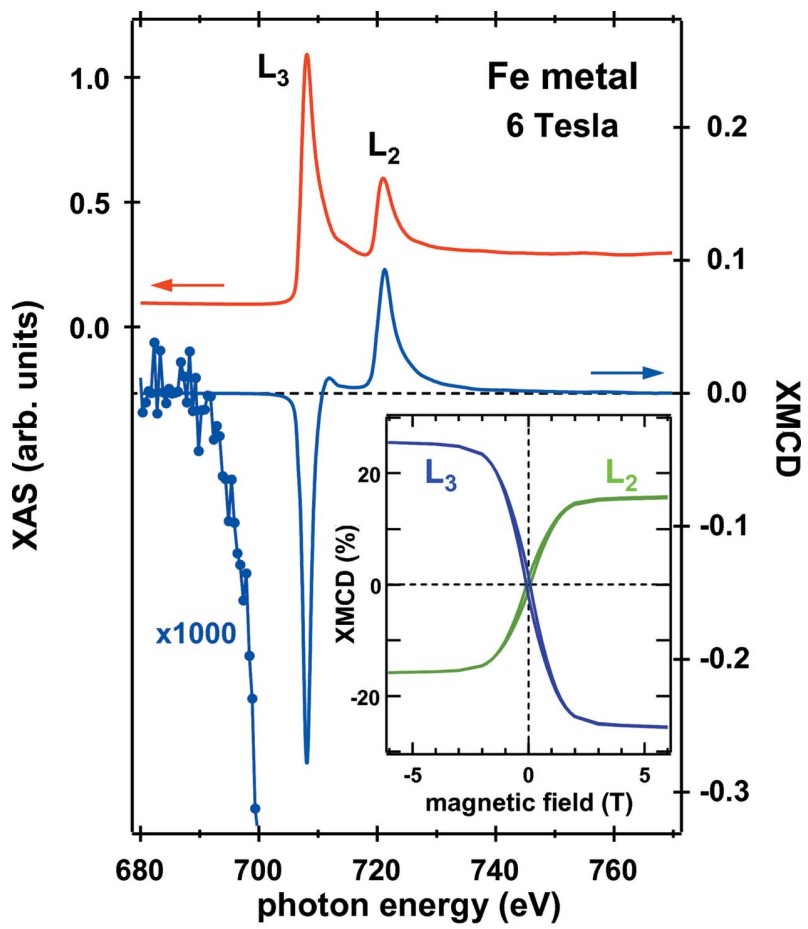

Figure 5

XAS and XMCD spectra at the $L_{2,3}$ edges of $\mathrm{Fe}$ metal at room temperature at $6 \mathrm{~T}$. The inset shows the XMCD hysteresis loops for the $L_{2,3}$ peak energies.

et al., 2001). In conventional XAS measurements without helicity switching, the noise level was suppressed to $\pm 0.02 \%$ or less of the averaged XAS intensity. The signal variations in the previous XMCD spectra were most likely due to a significant increase in heat load on the optical components caused by the linear polarization mode during the helicity switching.

The inset in Fig. 5 shows the XMCD asymmetry $\left[\left(\mu_{\mathrm{L}}-\mu_{\mathrm{R}}\right) /\right.$ $\left.\left(\mu_{\mathrm{L}}+\mu_{\mathrm{R}}\right)\right]$ recorded at the $L_{3}(708.1 \mathrm{eV})$ and $L_{2}(721.3 \mathrm{eV})$ edges while sweeping the applied magnetic field. No correction has been made to the curves. The XMCD asymmetry is known to be proportional to the element-specific magnetization projected along the incident photon wavevector (Chen et al., 1993). In addition, switching the field direction is equivalent to reversing the helicity of the incoming beam for ferromagnetic Fe. The magnetization curves indicate the same circular polarization rate $\left(\left|P_{\mathrm{c}}\right|\right)$ of the two beams as well as the full saturation of the Fe moment at $6 \mathrm{~T}$, because the XMCD signals for each helicity on reversing magnetization are the same.

The measured XMCD effect is consistent with the previous results of transmission experiments for magnetically saturated Fe films with a correction for the incomplete $P_{\mathrm{c}}$ (Chen et al., 1995). In addition, the XMCD asymmetry at $1.4 \mathrm{~T}$ is precisely equal to that obtained at BL25SU (Muro et al., 2005). The spectroscopy results clearly show that the helicity-switching operation of the twin-helical undulator is well suited for highprecision XMCD studies.

When the effective source point for LCP and RCP light is slightly different, this can transform into an energy difference 


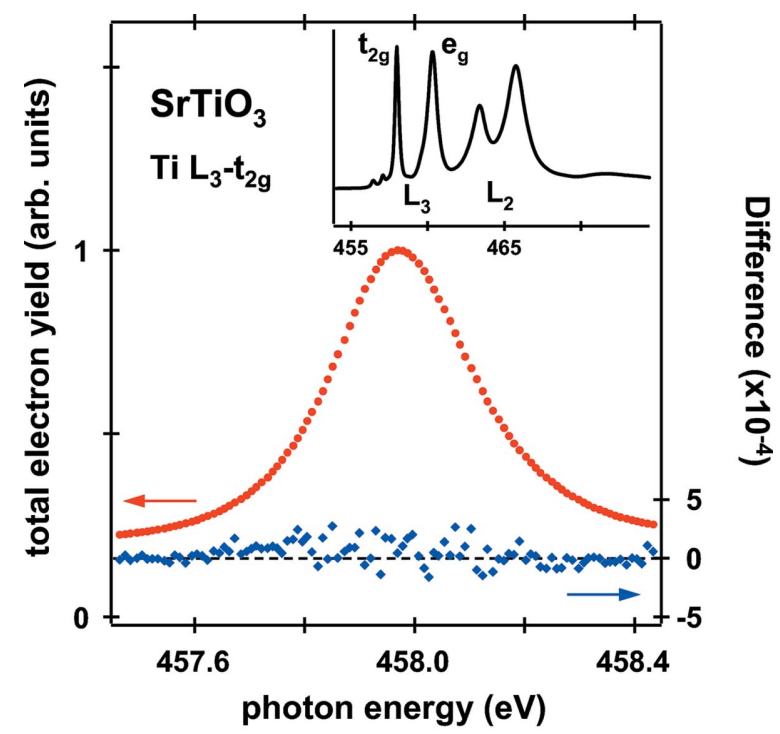

Figure 6

Absorption measurements for the $\mathrm{Ti} L_{3}-t_{2 \mathrm{~g}}$ transition of $\mathrm{SrTiO}_{3}$ at room temperature. Sum $\left(\mu_{\mathrm{L}}+\mu_{\mathrm{R}} ;\right.$ circles $)$ and difference ( $\mu_{\mathrm{L}}-\mu_{\mathrm{R}}$; diamonds) of the two intensities with opposite helicities. The inset shows the overview spectrum for the $\mathrm{Ti} L_{2,3}$ edges.

even for a given monochromator setting. The energy shift was examined by the measurement of the Ti $2 p_{3 / 2}-3 d\left(L_{3}-t_{2 g}\right)$ absorption spectra of a non-magnetic $\mathrm{SrTiO}_{3}$ sample as shown in Fig. 6. These spectra were taken at room temperature without applying a magnetic field for an energy resolution of $40 \mathrm{meV}$, in which both helicities in one cycle were used at every energy point. In the difference spectrum, no evidence of dichroic behavior is observed within the detection limit. An energy difference between the two beams will yield a derivative-shaped contribution to the difference spectrum. The energy difference is estimated to be not more than $2 \mathrm{meV}$ from the Voigt fit to the $\mu_{\mathrm{L}, \mathrm{R}}$ spectra.

\section{Application to a uranium compound}

As an example of investigations that were previously unapproachable, the XAS and XMCD spectra at the U $N_{4,5}$ edges in a paramagnetic $\beta$-US 2 single crystal measured along the $\langle 010\rangle$ direction at $20 \mathrm{~K}$ in an applied field of $10 \mathrm{~T}$ are shown in Fig. 7 (sample provided by Advanced Science Research Center, JAEA). These spectra prove U $5 f$ states through the dipole allowed $4 d_{5 / 2} \rightarrow 5 f_{5 / 2,7 / 2}\left(N_{5}\right)$ and $4 d_{3 / 2} \rightarrow$ $5 f_{5 / 2}\left(N_{4}\right)$ transitions. $\beta$-US $\mathrm{US}_{2}$ is a semiconductor and the $\mathrm{U}^{4+}\left(5 f^{2}\right)$ ionic scheme provides a good qualitative description of its magnetic behavior (Ikeda et al., 2009). So far, no detailed $\mathrm{XMCD}$ investigations of $\mathrm{U}$ compounds with formally $5 f^{2}$ ground state have been reported. The uranium magnetic moment projected along the photon wavevector is of the order of $0.1 \mu_{\mathrm{B}}$ under the experimental conditions, resulting in an XMCD variation of only $0.15 \%$ of the XAS intensity at the $N_{5}$ edge. In order to eliminate any experimental artifacts arising from system errors, the XMCD spectrum was measured for opposite orientations of the applied magnetic field and the resulting spectra were averaged. Each of the spectra was

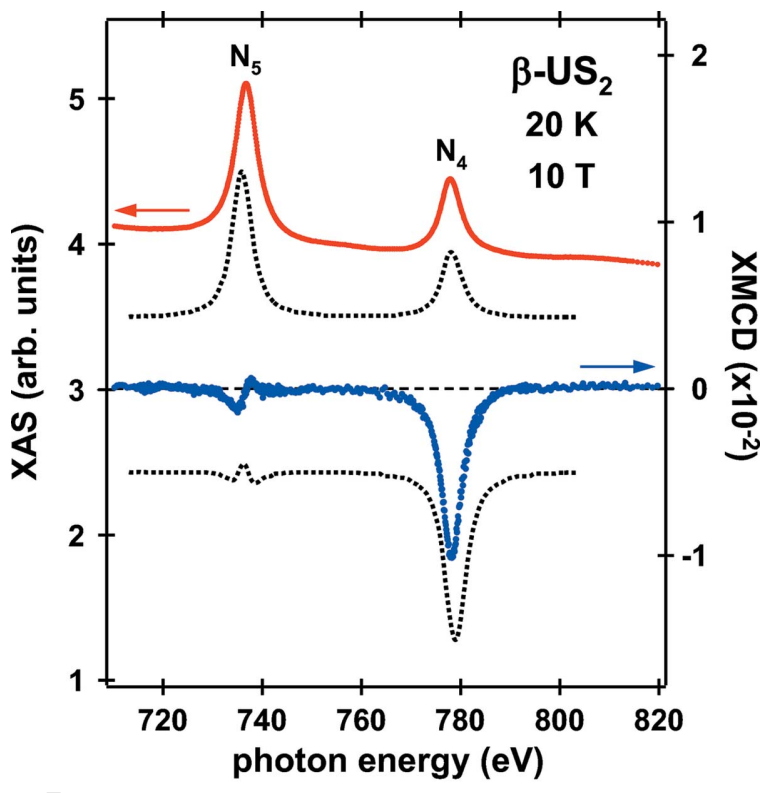

Figure 7

Uranium $N_{4,5}$ XAS and XMCD spectra obtained from paramagnetic $\beta$-US Ut $_{2}$ at $20 \mathrm{~K}$ for an external field of $10 \mathrm{~T}$ in comparison with atomic calculations for a $5 f^{2}$ ground-state configuration (dotted curves). The theoretical curves have been offset vertically for clarity.

acquired in two cycles of the helicity switching at every energy point with an energy resolution of $\sim 125 \mathrm{meV}$.

Application of the well known sum rules to the XMCD spectrum (Thole et al., 1992; Carra et al., 1993) provides valuable information on the $5 f$ magnetic moments. The sum rules relate the integrals of the XMCD signal over the $N_{5}$ edge ( $p$ ) and over both $N_{5}$ and $N_{4}$ edges $(q)$ to ground-state magnetic properties as

$$
\left\langle S_{\mathrm{e}}\right\rangle /\left\langle L_{z}\right\rangle=\left(\left\langle S_{z}\right\rangle+3\left\langle T_{z}\right\rangle\right) /\left\langle L_{z}\right\rangle=(5 p-3 q) / 4 q,
$$

where $\left\langle L_{z}\right\rangle,\left\langle S_{z}\right\rangle$ and $\left\langle T_{z}\right\rangle$ are the expectation values of the orbital angular momentum, spin angular momentum and the magnetic dipole term in the magnetization direction, respectively. $\left\langle S_{\mathrm{e}}\right\rangle$ is referred to as the effective spin. The ratio $\left\langle S_{\mathrm{e}}\right\rangle /$ $\left\langle L_{z}\right\rangle$ is instructive for the analysis of data on magnetically unsaturated samples and is independent of $P_{\mathrm{c}}$. From the measured XMCD spectrum, we derive $\left\langle S_{\mathrm{e}}\right\rangle /\left\langle L_{z}\right\rangle=-0.63 \pm$ 0.02 , which is in agreement with an atomic $5 f^{2}$ value of -0.66 calculated in intermediate coupling mechanisms for the angular momenta much better than that for $L S$ coupling of -0.46 (Collins et al., 1995; van der Laan \& Thole, 1996). Here we ignored in this analysis the so-called saturation effects in the total electron yield detection (Nakajima et al., 1999), which have not been investigated for $\mathrm{U} N_{4,5}$ edges both experimentally and theoretically. Hence, the transmission XMCD experiments of the samples in thin-film form are required. Such experiments, however, are virtually impossible in the BL23SU beamline, because the vaporization of uranium materials is not permitted in the SPring- 8 facility.

The sum-rule analysis uses only the integrated MCD intensities and ignores the information contained in the spectral shape. In Fig. 7, atomic calculations adopting the intermediate coupling scheme for the $\mathrm{U} 5 f^{2}$ ground state (van 
der Laan \& Thole, 1996) are compared. Although the experimental and theoretical curves possess the same qualitative features, there are some discrepancies in the XMCD structure at the $N_{5}$ edge. The apparent discrepancies are attributable to additional solid-state effects. In metallic uranium compounds, XMCD spectra at the $N_{5}$ and $M_{5}$ edges display a significant variation in shape (Antonov et al., 2008). Further analysis is currently under way (Takeda et al., 2012).

\section{Summary}

The new twin-helical undulator, with the capability of switching the photon helicity, installed in BL23SU at SPring-8 is now operational. This device has improved the XMCD sensitivity by an order of magnitude. The experiments performed on paramagnetic $\beta$-US $\mathrm{US}_{2}$ demonstrate the usefulness and promise of this technique. XMCD spectroscopy using lock-in detection methods is planned to allow more detailed studies. The new light source combined with on-going upgrades will elevate this beamline to an advanced instrumentation level and therefore greatly expand the scientific scope.

The authors are grateful for the continuous encouragement and support of Dr J. Mizuki and Dr K. Aoki (JAEA, Japan). We thank Dr S. Ikeda (University of Hyogo, Japan) and Dr Y. Haga (JAEA, Japan) for permission to include the data on $\beta$ $\mathrm{US}_{2}$. The XMCD measurements on $\beta$-US ${ }_{2}$ were performed under the approval of BL23SU at SPring-8 (Proposal No. 2011A3835) with the assistance of Dr T. Okane (JAEA, Japan). This work was financially supported in part by a Grant-in-Aid for Scientific Research on Innovative Areas 'Heavy Electrons' (No. 20102003) from the Ministry of Education, Culture, Sports, Science and Technology, Japan.

\section{References}

Agui, A., Yoshigoe, A., Nakatani, T., Matsushita, T., Saitoh, Y., Yokoya, A., Tanaka, H., Miyahara, Y., Shimada, T., Takeuchi, M., Bizen, T., Sasaki, S., Takao, M., Aoyagi, H., Kudo, T. P., Satoh, K., Wu, S., Hiramatsu, Y. \& Ohkuma, H. (2001). Rev. Sci. Instrum. 72, 3191-3197.

Antonov, V. N., Shpak, A. P. \& Yaresko, A. N. (2008). Low Temp. Phys. 34, 79-111.

Carra, P., Thole, B. T., Altarelli, M. \& Wang, X. (1993). Phys. Rev. Lett. 70, 694-697.

Chen, C. T., Idzerda, Y. U., Lin, H.-J., Meigs, G., Chaiken, A. \& Prinz, G. A. (1993). Phys. Rev. B, 48, 642-645.

Chen, C. T., Idzerda, Y. U., Lin, H., Smith, N. V., Meigs, G., Chaban, E., Ho, G. H., Pellegrin, E. \& Sette, F. (1995). Phys. Rev. Lett. 75, 152-155.

Collins, S. P., Laundy, D., Tang, C. C. \& van der Laan, G. (1995). J. Phys. Condens. Matter, 7, 9325-9341.

Freeland, J. W., Lang, J. C. Srajer, G., Winarski, R. Shu, D. \& Mills, D. M. (2002). Rev. Sci. Instrum. 73, 1408-1410.

Fujii, K., Shikazono, N. \& Yokoya, A. (2009). J. Phys. Chem. B, 113, 16007-16015.

Fujimori, S.-I., Saitoh, Y., Okane, T., Fujimori, A., Yamagami, H., Haga, Y., Yamamoto, E. \& Ōnuki, Y. (2007). Nat. Phys. 3, 618-622.

Funk, T., Deb, A., George, S. J., Wang, H. X. \& Cramer, S. P. (2005). Coord. Chem. Rev. 249, 3-30.
Hara, T., Shirasawa, K., Takeuchi, M., Seike, T., Saitoh, Y., Muro, T. \& Kitamura, H. (2003). Nucl. Instrum. Methods Phys. Res. A, 498, 496-502.

Hara, T., Tanaka, T., Tanabe, T., Maréchal, X.-M., Kumagai, K. \& Kitamura, H. (1998). J. Synchrotron Rad. 5, 426-427.

Hayashi, M., Narumi, Y., Nojiri, H., Nakamura, T., Hirono, T., Kinoshita, T., Kodama, K. \& Kindo, K. (2011). J. Electron Spectrosc. Relat. Phenom. 184, 338-341.

Hirono, T., Kimura, H., Muro, T., Saitoh, Y. \& Ishikawa, T. (2005). J. Electron Spectrosc. Relat. Phenom. 144-147, 1097-1099.

Ikeda, S., Sakai, H., Tateiwa, N., Matsuda, T. D., Aoki, D., Homma, Y., Yamamoto, E., Nakamura, A., Shiokawa, Y., Ota, Y., Sugiyama, K., Hagiwara, M., Kindo, K., Matsubayashi, K., Hedo, M., Uwatoko, Y., Haga, Y. \& Ōnuki, Y. (2009). J. Phys. Soc. Jpn, 78, 114704.

Kawasaki, I., Fujimori, S.-I., Takeda, Y., Okane, T., Yasui, A., Saitoh, Y., Yamagami, H., Haga, Y., Yamamoto, E. \& Ōnuki, Y. (2011). Phys. Rev. B, 83, 235121.

Kincaid, B. M. (1977). J. Appl. Phys. 48, 2684-2691.

Laan, G. van der \& Thole, B. T. (1996). Phys. Rev. B, 53, 14458-14469.

Muro, T., Nakamura, T., Matsushita, T., Kimura, H., Nakatani, T., Hirono, T., Kudo, T., Kobayashi, K., Saito, Y., Takeuchi, M., Hara, T., Shirasawa, K. \& Kitamura, H. (2005). J. Electron Spectrosc. Relat. Phenom. 144-147, 1101-1103.

Nakajima, R., Stöhr, J. \& Idzerda, Y. U. (1999). Phys. Rev. B, 59, 6421-6429.

Nakamura, T., Muro, T., Guo, F. Z., Matsushita, T., Wakita, T., Hirono, T., Takeuchi, Y. \& Kobayashi, K. (2005). J. Electron Spectrosc. Relat. Phenom. 144-147, 1035-1038.

Nakatani, T., Agui, A., Aoyagi, H., Matsushita, T., Takao, M., Takeuchi, M., Yoshigoe, A. \& Tanaka, H. (2005). Rev. Sci. Instrum. 76, 055105 .

Okamoto, J., Mamiya, K., Fujimori, S.-I., Okane, T., Saitoh, Y., Muramatsu, Y., Fujimori, A., Ishikawa, S. \& Takano, M. (2004). AIP Conf. Proc. 705, 1110-1113.

Oura, M., Nakamura, T., Takeuchi, T., Senba, Y., Ohashi, H., Shirasawa, K., Tanaka, T., Takeuchi, M., Furukawa, Y., Hirono, T., Ohata, T., Kitamura, H. \& Shin, S. (2007). J. Synchrotron Rad. 14, 483-486.

Oura, M., Sakurai, Y. \& Kitamura, H. (1998). J. Synchrotron Rad. 5, 606-608.

Pfleiderer, C. (2009). Rev. Mod. Phys. 81, 1551-1624.

Saitoh, Y., Nakatani, T., Matsushita, T., Agui, A., Yoshigoe, A., Teraoka, Y. \& Yokoya, A. (2001). Nucl. Instrum. Methods Phys. Res. A, 474, 253-258.

Sasaki, S. (1994). Nucl. Instrum. Methods Phys. Res. A, 347, 83-86.

Sawhney, K. J. S., Senf, F., Scheer, M., Schiifers, F., Bahrdt, J., Gaupp, A. \& Gudat, W. (1997). Nucl. Instrum. Methods Phys. Res. A, 390, $395-402$.

Sekiyama, A., et al. (2012). In preparation.

Shirasawa, K., Hara, T., Takeuchi, M., Hiraya, A. \& Kitamura, H. (2004). AIP Conf. Proc. 705, 191-194.

Suga, S., Shigemoto, A., Sekiyama, A., Imada, S., Yamasaki, A., Irizawa, A., Kasai, S., Saitoh, Y., Muro, T., Tomita, N., Nasu, K., Eisaki, H. \& Ueda, Y. (2004). Phys. Rev. B, 70, 155106.

Takeda, Y., et al. (2012). In preparation.

Takeda, Y., Kobayashi, M., Okane, T., Ohkochi, T., Okamoto, J., Saitoh, Y., Kobayashi, K., Yamagami, H., Fujimori, A., Tanaka, A., Okabayashi, J., Oshima, M., Ohya, S., Hai, P. N. \& Tanaka, M. (2008). Phys. Rev. Lett. 100, 247202.

Tanaka, T. \& Kitamura, H. (2001). J. Synchrotron Rad. 8, 1221-1228. Teraoka, Y. \& Yoshigoe, A. (2001). Appl. Surf. Sci. 169-170, 738741.

Thole, B. T., Carra, P., Sette, F. \& van der Laan, G. (1992). Phys. Rev. Lett. 70, 1943-1946.

Ukai, M., Yokoya, A., Nonaka, Y., Fujii, K. \& Saitoh, Y. (2009). Radiat. Phys. Chem. 78, 1202-1206.

Yokoya, A. \& Akamatsu, K. (2001). Nucl. Instrum. Methods Phys. Res. A, 467-468, 1333-1337. 